\title{
VARIATIONAL METHODS AND PERIODIC SOLUTIONS OF KIRCHHOFF-TYPE EQUATIONS. II
}

\author{
S. P. NOVIKOV
}

\section{INTRODUCTION}

In a previous paper [1], the author and Shmel'tser started the construction of an extended Lyusternik-Shnirelman-Morse theory for the study of single-valued and multivalued functionals on the space $\hat{\Omega}^{+}\left(M^{n}\right)$ of losed directed curves in a manifold $M^{n}$. The authors applied these methods to the classical problem (Kirchhoffs problem) about the free motion of a rigid body in an ideal incompressible liquid, where the fluid flows potentially and is at rest at infinity. From a modern point of view, Kirchhoff-type equations are defined by a Hamiltonian $H$ (coinciding with the classical energy) and Poisson brackets $\{$,$\} for functions in phase space (the$ dual space $L^{*}$ of the algebra $L$ of the group $E(3)$ of motions of the Euclidean space $\left.R^{3}\right)$. In the classical Kirchhoff case, we have

$$
\begin{gathered}
2 H=\sum_{i=1}^{3} a_{i i} M_{i}^{2}+2 \sum_{i, j=1}^{3} b_{i j}\left(\frac{p_{j} M_{i}+p_{i} M_{j}}{2}\right)+\sum_{i, j=1}^{3} c_{i j} p_{i} p_{j}>0, \\
\left\{M_{i}, M_{j}\right\}=\varepsilon_{i j k} M_{k}, \quad\left\{M_{i}, p_{j}\right\}=\varepsilon_{i j k} p_{k}, \quad\left\{p_{i}, p_{j}\right\}=0, \\
M_{i}=\left\{M_{i}, H\right\}, \quad \dot{p}_{i}=\left\{p_{i}, H\right\} .
\end{gathered}
$$

Here $M$ and $p$ are, respectively, the angular momentum and linear momentum in a moving coordinate system. Any quadratic form on $L^{*}$ is reducible to (1) by the action of $E(3)$. There exist universal Kirchhoff integrals such that

$$
\begin{gathered}
f_{1}=p^{2}=\sum p_{i}^{2}, \quad f_{2}=p s=\sum M_{i} p_{i}, \\
\left\{f_{l}, M_{i}\right\}=\left\{f_{l}, p_{i}\right\}=0, \quad l=1,2, i=1,2,3 .
\end{gathered}
$$

The present paper is a direct extension of [1]; a detailed list of references is given in [1].

\section{Nonpositivity of a Functional and Redundant Extremals}

By properties of the algebra $L$ of the group $E(3)$, on a level surface of the Kirchhoff integrals $(s, p)$ the Hamiltonian system (1) is reduced to a Lagrangian system on the sphere $S^{2}$. When $s \neq 0$, for topological reasons the Lagrangians are not defined on the whole sphere $S^{2}$ (they are defined only in domains $U_{\alpha} \subset$ $\left.S^{2}\right)$. Simultaneously, the collection of Lagrangians $L_{\alpha}$ in the domains $U_{\alpha}$ defines a "multivalued" functional $S$ (or $S_{E}$ ) on the space of closed curves. Here $\delta S_{E}$ turns

Date: Original article submitted January 15, 1981.

UDC. 517.9

L. D. Landau Institute for Theoretical Physics, Academy of Sciences of the USSR. Translated from Funktsional'nyi Analiz i Ego Prilozheniya, Vol. 15, No. 4, pp. 37-52, October-December, 1981. 
out to be a closed 1-form on the infinite-dimensional manifold of closed directed piecewise smooth curves on $S^{2}$. In this section the cases $s=0$ and $s \neq 0$ turn out to be essentially different (for $s=0$ the functionals $S$ and $S_{E}$ are single-valued). Both cases $(s=0$ and $s \neq 0)$ are characterized by the common property that the Lagrangians $\left(26^{\prime}\right)$ and $\left(26^{\prime \prime}\right)$ in [1] [see formulas (3) and (4) below] contain terms in $\dot{x}$. These terms (the "magnetic field") do not detract from the local Morse theory around a given curve depending only on the positivity of the matrix $\partial^{2} L / \partial \dot{x} \partial \dot{x}$. The main difference between Lagrangians of this type and functionals of length in Finsler geometry, where there is also no invariance under $t \rightarrow-t$, is the absence of strict positivity (and, or course, multivaluedness when $s \neq 0$ ). Although we have established the semiboundedness of $S_{E}(\gamma)$ for a special class of curves [1], nevertheless, it is also possible that for some curve $\gamma$ homotopic to zero (it must be homotopic to zero in a given domain $U_{\alpha}$ of single-valuedness of the Lagrangian in the multivalued case, where the "magnetic field" is a 2-form, which is not exact), the functional $S_{E}(\gamma)$ is less than zero. Since $S_{E}(\gamma)=0$ for one-point curves, the following lemma holds. ${ }^{1}$

Lemma 1. If there is a curve $\gamma$ homotopic to zero in a domain $U_{\alpha}$ such that $S_{E}(\gamma)<0$, then there are not less than two in the case of general position, and always not less than one "redundant" periodic orbits as well as the homologically nontrivial ones given by Theorem 1b of [1] and Theorem 2 of the present paper (see below).

Proof. This follows in a straightforward way from the fact that for all $E>\max U(x)$, the functional $S_{E}(\gamma)$ has a local minimum on the one-point curves. If $S_{E}(\gamma)<0$, then there is still a minimum, and a saddle in the case of general position, since for a minimum $\gamma_{\min }$ we must have $S_{E}\left(\gamma_{\min }\right)<0$. In the situation of Theorem $1 \mathrm{~b}$ of [1] and of Theorem 2 below, the saddle can degenerate (two saddles can merge into one degenerate one). This proves the lemma.

By [1], the Lagrangians corresponding to the Hamiltonian $H$ of Kirchhoff's problem have the form

where

$$
L(x, \dot{x})=\frac{1}{2} g_{l m} \dot{x}^{l} \dot{x}^{m}-A_{l} \dot{x}^{l}-U(x),
$$

$$
\begin{gathered}
A_{l} d x^{l}=s A_{l}^{(1)} d x^{l}+p A_{l}^{(2)} d x^{l}+s \sin \theta d \psi, \\
x^{1}=\theta, \quad x^{2}=\psi, \quad \xi_{1}=p_{\theta}, \quad \xi_{2}=p_{\psi}, \\
\sum p_{i}^{2}=p^{2}, \quad \sum M_{i} p_{i}=s p, \quad M_{i}=q_{i}+s p^{-1} p_{i}, \\
q_{1}=p_{\psi} \operatorname{tg} \theta \cos \psi-p_{\theta} \sin \psi, \quad q_{2}=p_{\psi} \operatorname{tg} \theta \sin \psi+p_{\theta} \cos \psi, \quad q_{3}=-p_{\psi}, \\
p_{2}=p \cos \theta \sin \psi, \quad p_{1}=p \cos \theta \cos \psi, \quad p_{3}=p \sin \theta, \\
g^{l m} \xi_{l} \xi_{m}=\sum a_{i i} q_{i}^{2}>0, \quad A_{l}^{(1)}=g_{l m} A_{1}^{m}, \quad A_{l}^{(2)}=g_{l m} A_{2}^{m}, \\
A_{1}^{m} \xi_{m}=\sum a_{i i} q_{i} p_{i} p^{-1}, \quad A_{2}^{m} \xi_{m}=\sum b_{i j}\left(q_{i} p_{i} p^{-1}+q_{j} p_{i} p^{-1}\right) / 2, \\
2 U(x)=s^{2}\left[\sum a_{i i} p_{i}^{2} p^{-2}-g_{l m} A_{1}^{l} A_{1}^{m}\right]+p^{2}\left[\sum c_{i j} p_{i} p_{j} p^{-2}-g_{l m} A_{2}^{l} A_{2}^{m}\right]+2 s p U_{12}, \\
U_{12}=\sum b_{i j} p_{i} p_{j} p^{-2}-g_{l m} A_{1}^{l} A_{2}^{m} .
\end{gathered}
$$

\footnotetext{
${ }^{1}$ Here we assume that $S_{E}=0$ on one component of single-point curves and $S_{E}>0$ on the second component; see the beginning of Sec. 2.
} 
By going over to the Lagrangian $L_{E}$ which is equivalent by "Maupertuis's principle," we obtain

$$
\begin{gathered}
L_{E} d t=\sqrt{2(E-U) g_{l m} d x^{l} d x^{m}}-s A_{l}^{(1)} d x^{l}-p A_{l}^{(2)} d x^{l}-s \sin \theta d \psi, \\
x^{1}=\theta, \quad x^{2}=\psi .
\end{gathered}
$$

In view of the gauge invariance of $L_{E}$ we can set $s^{2} E_{1}=E$ for $s \neq 0$ or $p^{2} E_{2}=E$ for $p \neq 0$, and extract from the Lagrangian the factor

$$
L_{E_{1}}^{(1)}=L_{E}|s|^{-1}, \quad L_{E_{2}}^{(2)}=L_{E} p^{-1}
$$

where the Lagrangians $L_{E_{1}}^{(1)}$ and $L_{E_{2}}^{(2)}$ have the same form but depend only on $s p^{-1}=$ $f_{2} f_{1}^{-1}$.

Lemma 2. Suppose that for a fixed $s p^{-1}, E_{1}$ or $E_{2}$ is decreasing. There is an $E_{10}$ (or $E_{20}$ ) depending on $s p^{-1}$ and on all the constants $a_{i i}, b_{i j}$, and $c_{i j}$ such that for $E>s^{2} E_{10}$ (or $\left.E>p^{2} E_{20}\right) S_{E}$ is strictly positive on the whole space of piecewise smooth closed directed non-self-intersecting curves on $S^{2}{ }^{2}$

Proof. Suppose that $s^{-2}(E-U) \rightarrow \infty$. Then the length of any curve is increasing. Whereas we showed earlier that the integral of a 1 -form $A_{l} d x$ is bounded in modulus for all non-self-intersecting curves. The proof follows at once from here.

We consider the Lagrangians $L$ and $L_{E}$ only for $E>\max U(\theta, \psi)$. There arises the question of the positivity of $L_{E}$ in the intervals

$$
\max V<E<s^{2} E_{10}, \quad \max U<E<p^{2} E_{20} .
$$

Lemma 3. There is a nontrivial domain of constants $\left(s p^{-1}, a_{i i}, b_{i j}, c_{i j}\right)$, where $S_{E}(\gamma)<0$ for certain $E>\max U$ and curves $\gamma$.

Proof. This is indicated by the following examples.

Example 1. Let $a_{11}=a_{22}=a_{33}, s \rightarrow \infty$ and $p=$ const. Then we obtain the Lagrangian (4), where after dividing $L_{E}$ by $s$, the potential tends to a constant as $s \rightarrow \infty$ :

$$
\begin{gathered}
U(\theta, \psi)=U_{0}+O(s) \sim s^{2}\left(\text { const }+O\left(s^{-1}\right)\right), \\
s^{-2} U_{0}=a_{11} \sum p_{i}^{2} p^{-2}=a_{11}, \\
s A_{1}^{l}+p A_{2}^{l}=s\left(A_{1}^{l}+O\left(s^{-1}\right)\right), \\
A_{1}^{l} \xi_{l}=\sum a_{i i} q_{i} p_{i} p^{-1}=a_{11} p^{-1} \quad\left(\sum q_{i} p_{i}\right)=0 .
\end{gathered}
$$

In view of (7), $(E-U) g_{l m}$ can be made arbitrarily small as $E \rightarrow U_{0}$ and $s \rightarrow \infty$. Deduction: For any curve $\gamma$ on $S^{2}$ there is an $E_{1}=E s^{-2}$ sufficiently close to $S^{-2} U_{0}=$ const that $S_{E}(\gamma)<0(s \rightarrow \infty)$.

Example 2. Let $b_{i j}=\gamma a_{i i} \delta_{i j}$ and $c_{i j}=\left(\lambda+a_{i i}\right) b_{i i}^{2} a_{i i}^{-2} \delta_{i j}$; we set $s p^{-1}=b_{i i} a_{i i}^{-1}$. Then we obtain the potential $U=$ const in the Lagrangian $L$. Again, as in Example 1 , for any curve $\gamma$ homotopic to zero in a domain $U_{\alpha}$ on $S^{2}$, along which the integral of $\left(A_{l} d x^{l}\right)$ is nonzero, we obtain $S_{E}(\gamma)<0$ if $E \rightarrow U=U_{0}$.

\footnotetext{
${ }^{2}$ See footnote previous page.
} 
Next we try to look for the "small" test curves $\gamma$ for which $S_{E}(\gamma)<0$. We consider the point $x_{0}$ where the potential $U$ attains a maximum

$$
\begin{gathered}
U\left(\theta_{0}, \psi_{0}\right)=U_{0}=\max U, \quad \operatorname{grad} U\left(\theta_{0}, \psi_{0}\right)=0, \\
{\left[\partial^{2} U / \partial x^{i} \partial x^{j}\right] d x^{i} d x^{j}<0 .}
\end{gathered}
$$

We choose a coordinate system about this point so that at $x=x_{0}$

$$
2 d^{2}\left(U_{0}-U\right)=\lambda^{2}(d x)^{2}+\mu^{2}(d y)^{2}, \quad \lambda^{2}=\lambda_{\max } \geqslant \mu^{2}, \dot{g}_{l m}=\delta_{l m}=\dot{g}^{l m} .
$$

We select a test curve $\gamma=\gamma_{\varepsilon}$ in the tangent space at $x_{0}$ in the form of a square of side $2 \varepsilon$ with center at $x_{0}$ and sides parallel to the principal axes $x, y$ of the form $d^{2}\left(U_{0}-U\right)$. For small $\varepsilon \rightarrow 0$ we can project $\gamma=\gamma_{\varepsilon}$ from the tangent space onto the sphere $S^{2}$.

Lemma 4. As $(\lambda, \mu)$ varies and for a sufficiently small $\varepsilon \rightarrow 0$ the quantity $l=$ $\oint \sqrt{2\left(U_{0}-U\right) g_{l m} d x^{l} d x_{m}}$ satisfies the inequalities $6 \lambda_{\max } \varepsilon^{2}<l<9.2 \varepsilon^{2} \lambda_{\max }$ if $\lambda_{\max }=\lambda^{2} \geqslant \mu^{2}$.

Proof. This is by a direct calculation with the following asymptotic equality holding as $\varepsilon \rightarrow 0$ :

$$
\begin{gathered}
L_{U_{0}}=L_{E} \sim \sqrt{\left(\lambda^{2} x^{2}+\mu^{2} y^{2}\right)\left(d x^{2}+d y^{2}\right)}-A_{l} d x^{l}, \\
l \sim 4 \lambda \int_{0}^{\varepsilon}\left\{\sqrt{\varepsilon^{2}+\frac{\mu^{2}}{\lambda^{2}} y^{2}} d y+\sqrt{x^{2}+\frac{\mu^{2}}{\lambda^{2}} \varepsilon^{2}} d x\right\} .
\end{gathered}
$$

Theorem 1. a) We consider on $S^{2}$ a point $x_{0}$ of maximum potential $U(\theta, \psi)$ of the Kirchhoff problem (1) for certain constants $a_{i i}, b_{i j}, c_{i j}$, and $s p^{-1}$, and the maximum eigenvalue $\lambda_{\max }$ of the form $2 d^{2}\left(U_{0}-U\right)$ :

$$
\operatorname{det}\left(2 g^{k m} \frac{\partial^{2}\left(U_{0}-U\right)}{\partial x^{l} \partial x^{m}}-\lambda_{\max } \delta_{l}^{k}\right)=0 .
$$

If for the effective magnetic field $H=H_{12}\left(x_{0}\right)$

$$
9.2 \lambda_{\max }<4\left|H_{12}\left(x_{0}\right)\right| \sqrt{\operatorname{det} g^{l m}},
$$

then for the above test curve, $S_{U_{0}}\left(\gamma_{\varepsilon}\right)<0, \varepsilon \rightarrow 0$. Here $H_{12}=\partial A_{1} / \partial x^{2}-\partial A_{2} / \partial x^{1}$,

b) If $U=$ const, then this is true for any curve $\gamma$ provided that $E$ is sufficiently close to $U_{0}$ (and the flux of the field $H_{12}$ through the area is nonzero).

Corollary. For both of these cases there are not less than two "redundant" periodic orbits for all $s$ and for all energies $E$ in some nonzero interval $\max U=U_{0}<E<$ $U_{1}$.

(The term "redundant" is used for a comparison with the minimally necessary number arising from the homologies.) The fulfillment of (10) depends on the constants $\left(a_{i i}, b_{i j}, c_{i j}, s p^{-1}\right)$.

Proof of Theorem 1. This follows immediately from the preceding lemmas and the following observation: inequality (10) is simply an invariant form of notation for the fact that the value of the integral along $\gamma_{\varepsilon}$ of the linear (with respect to velocity) term in $L_{U_{0}}$, which is $\approx 4 \varepsilon^{2} H_{12}$ for components in the principal axes, is greater in modulus than the integral of the kinetic energy on the "critical" level surface $E=$ $U_{0}$. By a proper choice of the direction of the curve $\gamma=\gamma_{\varepsilon}$ we obtain $S_{E}(\gamma)<0$. By fixing $\gamma$ we can decrease $E$ while preserving the inequality $S_{E}(\gamma)<0$. 
Remark. Theorem 1 and its corollary remain valid for the motion of a charged particle over an ideally conducting metallic sphere $S^{2} \subset \mathbb{R}^{3}$ under some embedding with a metric $g_{l m}$, in the external magnetic field $H_{12}$ in the interval (6) in terms of the energy, if either the potential is trivial but the energy is less than some threshold $E<\tilde{E}_{1}$, or the magnitude of the field $H_{12}$ at the point of maximum potential (if it is nontrivial) satisfies (10). Here the saddle trajectory $\gamma_{1}$, where $S_{E}\left(\gamma_{1}\right)>0$, lies very close to the maximum point $x_{0}$ (and is created" from this point under a bifurcation with respect to the parameter $E$ ), whereas the minimal trajectory $\gamma_{2}$, where $S\left(\gamma_{2}\right)<0$, can lie very far away from $x_{0}$ and does not undergo bifurcation as $E$ passes through $U_{0}$.

It is of interest to try by analogy with [2] (Chap. 6) to analyze also the case $E<\max U$, taking into account the property of variational problems investigated here and resulting from the "magnetic" terms in the Lagrangian. One should bear in mind that when $E<\max U$ the difficulties connected with the effect of the multivaluedness of the action and the Lagrangians disappear, since any 2-form is exact on $S^{2}$ with a punctured neighborhood of a point. Questions relating to the nonpositivity of the functional that result from the "magnetic" terms present serious difficulties.

2. Periodic Motions in the Kirchhoff Problem when $f_{2} \neq 0$, and in the Problem of the Motion of a Heavy Rigid Body in an Axisymmetric Gravitational Field $(j \neq 0)$

We consider a multivalued functional or 1-form $\delta S_{E}(\gamma)$ only on the space $\hat{\Omega}_{1}^{+}$of directed non-self-intersecting curves $\gamma \subset S^{2}$, in accordance with [1]. Since this space is simply connected [it contracts to $S^{2} \times I(-1,1)$, where both edges of the interval $I$ correspond to one point curves], the infinite-dimensional 1-form $\delta S_{E}$ defines on $\hat{\Omega}_{1}^{+}\left(S^{2}\right)$ a single-valued function (functional) $S_{E}$ such that $S_{E}\left(\gamma_{0}\right)=0$, where the $\gamma_{0}$ are one-point curves. But in constructing the natural compactification $K$ of $\hat{\Omega}_{1}^{+}$ by one-point curves $K_{0}$ by means of the functional $S_{E}$, we approach $K_{0}$ "from two sides" and with two values of $S_{E}$. One of these is zero, while the second is a positive number. Therefore, $K_{0}$ lies on the boundary of $K$ in two copies of $K_{0}^{+} \cup K_{0}^{-}$.

Now we state explicitly an essentially self-evident property of a multivalued functional $S_{E}$ on the space $\hat{\Omega}_{1}^{+}$; the use of this property was overlooked in [1]: on any sheet of the covering of the space of directed closed curves, the complete inverse image of the one point curves is a local minimum of $S_{E}$ (which is single-valued on the covering). It follows directly from here that under the map $S^{2} \times I \rightarrow K$ of the embedding of the set of plane sections, where the boundary $S^{2} \times 1 \cup S^{2} \times(-1)=B$ maps into one-point curves $K_{0}^{+} \cup K_{0}^{-} \subset K$, the image of both basic cycles modulo the boundary from the groups $H_{*}\left(S^{2} \times I, B\right)$ of both one- and three-dimensional cycles, in a sufficiently small neighborhood of the edges always lies (in terms of the value of $S_{E}$ ) "above" the one-point curves. This property is preserved under any gradient-similar deformation fixed at the edges that is pointwise "below" in terms of the levels of the functional. Hence, both cycles "hang" at nontrivial critical points with the levels of the functional "above" both of the one-point curves. Thus, we obtain the ultimate general result. 
Theorem 2. Let an arbitrary Kirchhoff type Hamiltonian of the following form be defined on the phase space $L^{*}$ dual to the algebra $L$ of the group $E(3)$ :

$$
2 H=\sum a_{i j}(p) M_{i} M_{j}+2 \sum b_{i}(p) M_{i}+V(p),
$$

where the form $a_{i j}(p)$ is positive for all $p$. Then for any fixed values of the Kirchhoff integrals $(p \neq 0, s)$, and for any energy $E$ greater than some threshold, $E>E_{0}(s, p)$, the system has not less than two non-self-intersecting periodic trajectories for which the value of the functional $S_{E}$ is greater than $4 \pi|s|$. After reducing system (11) to a Lagrangian system on $S^{2}$ with fixed $(s, p \neq 0), E_{0}(s, p)$ coincides with the maximum of the potential.

Proof. This is given above together with the observation that the reduction of system (11) to a Lagrangian system leads in an obvious way to a Lagrangian of the form (3), (4) with a positive kinetic energy (the metric on $S^{2}$ ) depending only on the form $a_{i j}$. The value of $S_{E}$ on the second one-point component $K_{0}^{-}$is (see [1])

$$
4 \pi|s|=\left|\iint_{S^{2}} \Omega\right|=\left|\iint_{S^{2}} s \cos \theta d \theta \wedge d \psi\right| .
$$

In the Kirchhoff problem we always have $a_{i j}>0$, the whole Hamiltonian $H$ is a positive quadratic form. We obtain the following corollary.

Corollary 1. The Kirchhoff equations (1) always have not less than two periodic non-self-intersecting trajectories for any energy $E>\max U(x)$.

The problem of the motion of a heavy rigid body about a fixed point in an axisymmetric gravitational field can be reduced to a problem in phase space $L^{*}$ with a Hamiltonian of the form $(11)^{3}$ :

$$
2 H=\sum a_{i i} M_{i}^{2}+2 V\left(R^{i} p_{i}\right), \quad \sum p_{i}^{2}=1
$$

where $V(z)$ is the gravitational potential and $R=\left(R^{i}\right)$ is the center of mass. For example, the classical case $V=-m g z$ is nontrivial. We obtain the following corollary.

Corollary 2. For any value of the "area integral" (corresponding to $f_{2}$ when this problem is embedded the phase space $\left.L^{*}\right)$ and any value $E>E_{0}(s, p)$, there are not less than two periodic motions (for $f=0$ see [2, Chap. 6]).

Now we turn our attention to the following interesting situation: the Kirchhoff Hamiltonian when $a_{i 3}=b_{i 3}=c_{i 3}=0(i \neq 3)$ (and the Hamiltonian of a heavy rigid body in an axisymmetric gravitational field, and $R^{1}=R^{2}=0$ always admit a symmetry, viz., a reflection in the $p_{3}$ axis (see Sec. 5). Consequently, in this case we can apply the reasoning of Sec. 5: there are two periodic orbits among the plane sections of the sphere $S^{2}$ orthogonal to the $p_{3}$ axis (one for each direction with any value of the area integral $j$ or $f_{2}$ ). These orbits can probably be found explicitly; the author assumes that they were known to early investigators, in any case for a heavy rigid body in the potential field $V=-m g z$ (it cannot be otherwise!).

\footnotetext{
${ }^{3}$ It is curious that in this case the $p_{i}$ do not have the physical meaning of momentum, in contrast to the Kirchhoff problem. If $A$ is a transformation from a fixed coordinate system to a rotating one, and $e_{3}$ is the unit vector along the $z$ axis, then $p=A\left(e_{3}\right)$. It can be seen that $p$ is nondimensional.
} 
The appearance of "redundant" orbits must be sought by the method of Sec. 1 [new orbits found by this method turn out to be plane sections if the maximum of the potential $U(\theta, \psi)$ lies in a strip]. Thus, for $j \neq 0$ or $f_{2} \neq 0$, when there is an axial reflection (a symmetry of the second order), the extended calculus of variations "in the large" (Lyusternik-Shnirelman-Morse theory) ensures that the minimal number of critical points of the functional demanded by homology theory is gathered entirely from plane sections. An algorithm for finding these solutions is as follows: 1) we integrate the Lagrangian $L_{E}$ overplane sections of a given direction that are orthogonal to $p_{3} ; 2$ ) then we regard the result of the integration as a function of $p_{3}$ and find its extremum with respect to $p_{3}$.

In the case of an even potential $V\left(z^{2}\right)$ and $R^{1}=R^{2}=0$, we obtain a Hamiltonian $H$ which is invariant under the group $\mathbb{Z}_{2} \times \mathbb{Z}_{2}$ of all reflections in orthogonal axes; see also Sec. 5, Example 2. In this case we have trivially six periodic trajectories of the form of plane rotations for all values of $j .{ }^{4}$

\section{Variational Problems on the Space of All Curves for Compact Two-Dimensional Manifolds $(E \mapsto \infty)$}

We use the fact, proved in Lemma 2, that $S_{E}(\gamma)$ is positive for some $E>E_{0}$ for closed non-self-intersecting curves on a compact two-dimensional manifold $M^{2}$ of some homotopy class, and for any direction. Hence, it follows that for $E>E_{0}$ the action $S_{E}(\gamma)$ is positive for all piecewise-smooth closed curves, since a curve in general position can be easily broken down into non-self-intersecting curves. Thus, we have the following lemma.

Lemma 5. For Lagrangians of the form (3), (4) with a positive metric $g_{l m}$ on a compact manifold $M^{2}$, there is an energy "threshold" $E_{0}$ such that for $E>E_{0}$, $S_{E}(\gamma)$ is positive for any piecewise-smooth directed curve.

Deduction. For sufficiently large energies $E \geqslant E_{0}$, any pair of points $x_{1} \in M^{2}$, $x_{2} \in M^{2}$ can be joined by at least one extremal of the Lagrangian $L_{E}$ for any direction $x_{1} \rightarrow x_{2}$ or $x_{2} \rightarrow x_{1}$.

Note that in a problem with two end points it is not possible, in principle, to work with spaces of non-self-intersecting curves, in contrast to a periodic problem on two-dimensional manifolds.

\section{Some Examples of Variational Problems in a Magnetic Field on Complete Riemannian Manifolds (Open and Compact)}

Before turning to results of a general nature, we discuss some useful examples.

I. We consider the Lobachevskii plane $L^{2}$ with, e.g., a standard metric, and a magnetic field, i.e., a 2 -form $H_{12} d \sigma$ ( $d \sigma$ is an element of area), and possibly a potential field $V(x, y)$ that are invariant under some discrete group of motions $\Gamma$ (from a compact fundamental domain). First we suppose that $V \equiv 0$ and $H_{12}=$ $H=$ const. We consider an associated pair of Lagrangians in $L^{2}(y>0)$

$$
\begin{gathered}
L=\frac{m l_{0}^{2}\left(\dot{x}^{2}+\dot{y}^{2}\right)}{2 y^{2}}+\frac{e H l_{0}^{2}}{c y} \dot{x}, \\
L_{E} d t=\sqrt{2 E m l_{0}^{2} y^{-2}\left(d x^{2}+d y^{2}\right)}+\frac{e H}{c y} l_{0}^{2} d x,
\end{gathered}
$$

\footnotetext{
${ }^{4}$ If $V=V\left(z^{2}\right)$ and $R^{1}=0$, then we have the group $\mathbb{Z}_{2}$ of reflections in the $p_{1}$ axis.
} 
where $l_{0}$ is the "radius of curvature." An integration leads without difficulty to the fact that starting from some threshold of energy $E$, the trajectories recede to infinity (in absolute magnitude), and for a fixed $E$ any pair of points can be joined by an extremal. Thus, the condition for the motion to be infinite has the form

$$
\omega=\frac{e H}{m c}, \quad \omega<\frac{2 E}{m l_{0}^{2}} .
$$

The reason for this is intuitively obvious: it is known that for large circles in $L^{2}$ the length and area have the same order of magnitude. Whichever of these is greater when the Lagrangian $\left(13^{\prime \prime}\right)$ is integrated along the curve is decided by the constants $E, H$, and $l_{0}(e, c$, and $m$ are fixed); see the discussion at the end of Sec. 2 about a magnetic field on a plane.

Now we consider a field $H(x, y)$ invariant under a discrete group $\Gamma$ with a compact factor, and a mean field $\bar{H}$, where the form $(H(x, y)-\bar{H}) d x \wedge d y y^{-2}$ is exact on the compact factor-space $L^{2} / \Gamma=M^{2}$. The following holds.

Assertion. There is a unique constant $A>0$ such that for $4 \omega^{2}<E m^{-1} l_{2}^{-2}$, $\omega=e \bar{H}_{12} m^{-1} c^{-1}$, the functional $S_{E}(\gamma)$ is semibounded on the space of piecewisesmooth directed curves (self-intersecting or not, and of any orientation).

Consequently, in this case, any pair of points of $L^{2}$ can be joined by an extremal with a fixed $E$; there are necessarily extremals periodic on $L^{2} / \Gamma$ with any homotopy class, apart from the trivial one. This is true also for metrics periodic under $\Gamma$, and also in a periodic potential field $U$ if $E>\max U$.

II. We have a close situation for the motion of charged particles over a closed metal surface (of any shape) located in an external magnetic field $H_{i j}$. In this case the assertion simplifies: the mean field $\bar{H}_{i j}$ is always zero. Another case is more interesting.

The metal surface can be $M^{2} \subset \mathbb{R}^{3}$, which outside a compact domain coincides with the plane $\mathbb{R}^{2} \subset \mathbb{R}^{3}$, located in a (for example) constant magnetic field $H_{i j}$ in $\mathbb{R}^{3}$. The surface $M^{2}$ is distorted in the finite part distinct from the plane, and can have handles, i.e., a nontrivial topology (genus $g>0$ ). We consider the action functional $S_{E}(\gamma)$ on the space of closed directed non-self-intersecting curves $\gamma$ homotopic to a large circle lying in the "exterior" region, where $M^{2}=\mathbb{R}^{2}$, with a direction opposite to the motion in circular orbits on the plane $\mathbb{R}^{2}$ in a constant field $H_{i j}$. For a genus $g \geqslant 1$ these curves are not homotopic to zero (there are no point curves among them). By analogy with part c) of Theorem 2 of [1], we see that $S_{E}(\gamma)$ is semibounded.

Assertion. In this situation there must be a periodic orbit directed opposite to the circular trajectories of the motion on $\mathbb{R}^{2}$ in the field $H_{i j}$. These trajectories "hang" on the handles of the surface $M^{2} \subset \mathbb{R}^{3}$, where it differs from the plane $\mathbb{R}^{2}$.

III. Now we consider a two-dimensional problem of the motion of particles in the $(x, y)$ plane in a doubly periodic external magnetic field $H_{12}(x, y)$ directed along the $\mathrm{z}$ axis with a nonzero flux through an elementary cell $K$

$$
\bar{H}_{12}=\frac{1}{|K|} \iint_{K} H_{12} d x \wedge d y \neq 0
$$

This problem (partly discussed in [1]) can be interpreted as a Hamiltonian motion on the torus $T^{2}$ with a trivial Hamiltonian $H=m\left(\dot{x}^{2}+\dot{y}^{2}\right) / 2=p^{2} / 2 m$, but a 
nontrivial Poisson bracket [1]

(16) $\{x, y\}=\left\{x, p_{y}\right\}=\left\{y, p_{x}\right\}=0, \quad\left\{x, p_{x}\right\}=\left\{y, p_{y}\right\}=1, \quad\left\{p_{x}, p_{y}\right\}=H_{12}(x, y)$.

A Lagrangian of the form (3), (4) arises in the usual way. We take the space of closed non-self-intersecting piecewise-smooth curves described in the direction of rotation of the circular orbits in a constant field $\bar{H}_{12}$. For large (in area compared with length) trajectories $\gamma$, the action $S(\gamma)<0$. By considering a 1-parameter family of curves emanating from one-point curves to infinitely wide ones, and by deforming them "below" in terms of a gradient with a fixed initial point, it is obvious that we shall "sit" at the critical points of index 1 . Thus, the following theorem holds.

Theorem 3. For every energy $E>0$ there is at least one periodic non-selfintersecting orbit in $\mathbb{R}^{2}$ if the magnetic field $H_{12}(x, y)$ directed along the $z$ axis is double periodic, and the mean field $\bar{H}_{12}$ is nonzero. This periodic orbit turns in the same direction as in a constant field. ${ }^{5}$

Remark. This result is also true when the magnetic field is such that the mean $\bar{H}_{R}$ over a disk of large radius $R$ does not tend to zero with $R$ (or it tends slowly, slower than $R^{-1}$ ) while keeping the same sign as $R \rightarrow \infty$.

IV. Now we turn to the formulation of problems of a general nature connected with the essentially multivalued functionals defined in [1]. Let $M^{n}$ be an arbitrary complete Riemannian manifold with a metric $g_{i j}$. [If the potential $U(x)$ is such that $E>\max U(x)$ for a given energy, then it can be eliminated by a reduction to a new metric $\tilde{g}_{i j}^{E}=(E-U(x)) g_{i j}$. We require that Maupertuis's metric $\tilde{g}_{i j}^{E}$ is complete. Hence we can assume that $U \equiv 0$.] Suppose that there is defined a closed nonexact 2-form (a "magnetic field")

$$
0 \neq\left[H_{i j} d x^{i} \wedge d x^{j}\right] \in H^{2}\left(M^{n}, \mathbb{R}\right) .
$$

As usual, let $\hat{\Omega}^{+}\left(M^{n}\right)$ denote the space of all piecewise-smooth directed closed curves on $M^{n}$, and $\hat{\Omega}^{+}\left(M^{n}, x_{1}, x_{2}\right)$ denote the space of directed paths going from $x_{1}$ to $x_{2}$. There is a natural Serre fibering $\hat{\Omega}^{+}\left(M^{n}\right) \stackrel{\pi}{\rightarrow} M^{n}$ with a fiber $\hat{\Omega}^{+}\left(M^{n}, x_{1}, x_{2}\right)=$ $\Omega^{+}\left(M^{n}, x_{1}\right)$, where to a closed path $\gamma(t)$ there corresponds $\pi(\gamma)=\gamma(0)\left[\gamma(0) \in M^{n}\right.$ is the value at 0]. There is a section $q: M^{n} \rightarrow \hat{\Omega}^{+}\left(M^{n}\right)$ consisting of one-point curves $M^{n} \subset \hat{\Omega}^{+}\left(M^{n}\right)$. In the nonsimple connected case $\pi_{1}\left(M^{n}\right) \neq 0$, we must assume that $\hat{\Omega}^{+}\left(M^{n}\right)$ is the space of curves homotopic to zero in $M^{n}$; we denote by $\tilde{M}^{n}$ the universal covering of $M^{n}$. Obviously, $H_{1}\left(\hat{\Omega}^{+}\left(M^{n}\right), M^{n} ; \mathbb{R}\right)=H_{2}\left(\tilde{M}^{n}, \mathbb{R}\right)$. There are two cases:

a) if $\pi_{1}\left(M^{n}\right)=0$, then $\tilde{M}^{n}=M^{n}$;

b) if $\pi_{1}\left(M^{n}\right) \neq 0$, then it is possible that $H_{2}\left(M^{n}, R\right) \neq 0$ but $H_{2}\left(\tilde{M}^{n}, \mathbb{R}\right)=0$ (e.g., when $M^{n}=T^{n}$ ).

We assume that in the case being studied $\pi_{1}\left(M^{n}\right)=0$, or more generally, although $\pi_{1}\left(M^{n}\right) \neq 0$, the magnetic field has a cohomology class coining from $H_{2}\left(\tilde{M}^{n}\right) \rightarrow H_{2}\left(M^{n}\right)$. The second case is reduced to the first by the substitution

\footnotetext{
${ }^{5}$ The one-point curves form the torus $T^{2}$. Similarly, each cycle $z_{i} \in H_{i}\left(T^{2}\right)$ in general position generates across a "saddle point" a critical point of index $i+1$. Thus, we obtain four periodic orbits for each energy $E>0$ (they are all non-self-intersecting). It should be borne in mind that this problem cannot originate from the physics of a rigid body: the length of the path of a classical particle in a crystal is very small.
} 
$M^{n} \rightarrow \tilde{M}^{n}$. Hence we can assume from the beginning that $\pi_{1}\left(M^{n}\right)=0$. In this case, by [1] we have in any domain $U_{\alpha} \subset M^{n}$ a Lagrangian $L_{\alpha}$ such that the 2 -form of the magnetic field $H_{i j} d x^{i} \wedge d x^{j}$ is exact in $d \omega_{\alpha}=d\left(A_{i} d x^{i}\right)=H_{i j} d x^{i} \wedge d x^{j}$. Let

$$
L_{\alpha} d t=\omega_{\alpha}-H d t+\sum p_{i} d x^{i}
$$

where $H$ is the Hamiltonian generated by the metric

$$
2 H=g^{i j} p_{i} p_{j},
$$

$x^{i}$ are local coordinates and the $p_{i}$ denote momenta. The Lagrangian $L_{\alpha}$ has the form

$$
L_{\alpha}=\frac{1}{2} g_{i j} \dot{x}^{i} \dot{x}^{j}=A_{i} \dot{x}^{i}
$$

It is obvious that in our case the positivity condition holds

$$
\xi^{i} \xi^{j} \frac{\partial^{2} L_{\alpha}}{\partial \dot{x}^{i} \partial \dot{x}^{j}}>0
$$

According to [1] there is a many-valued functional $S_{E}(\gamma)$ on the space $\hat{\Omega}^{+}\left(M^{n}\right)$, i.e., $\delta S_{E}$ is a closed 1-form and thereby a single-valued function on some nontrivial minimal covering $f: \hat{\Omega}^{+} \rightarrow \hat{\Omega}^{+}\left(M^{n}\right)$ (of infinitely many sheets). The covering $f$ is regular with the free Abelian monodromy group $\mathbb{Z} \times \cdots \times \mathbb{Z}$. We denote the generating monodromy groups by $T_{1}, \ldots, T_{k}$. The number $k$ of them is determined by general considerations as follows: we need to choose a basis $z_{1}, \ldots, z_{l}$ in the group $H_{1}\left(\hat{\Omega}^{+}\left(M^{n}\right), \mathbb{Z}\right)$ (mod torsion) and consider "integrals" of the 1 -form $\delta S_{E}$ along the paths $z_{1}, \ldots, z_{l}$ in the space $\hat{\Omega}^{+}\left(M^{n}\right)$ ["scalar products" $\left(\delta S_{E}, z_{j}\right)=\kappa_{j}$. As always, the number of generators $k$ of the monodromy group of the minimal covering of $\hat{\hat{\Omega}}^{+}$that turns $\delta S_{E}$ into an exact 1 -form on $\hat{\hat{\Omega}}^{+}$, is equal to the rank of the collection of real numbers $\left(\kappa_{1}, \ldots, \kappa_{l}\right)$ over the integers. Next it is convenient to assume, making integer changes of the basis, that $\kappa_{j}=0$ for $j>k$.

Note that the quantities $\kappa_{j}$ are exactly the set of integrals of the magnetic field, i.e., of the 2-form $H_{i j} d x^{i} \wedge d x^{j}$ over the base of the cycles in the group $H_{2}\left(M^{n}, \mathbb{Z}\right) /$ Torsion $=H_{1}\left(\hat{\Omega}^{+}\left(M^{n}\right), M^{n}, \mathbb{Z}\right) /$ Torsion. Thus, for simply connected manifolds $M^{n}$, the homologically nontrivial magnetic field generates a many-valued functional, where the monodromy group of the minimal covering eliminating the multivaluedness, is defined by the integrals of the field over the basic integral 2cycles. If the magnetic field yields integer-valued integrals $\kappa_{j}$, then the covering of $\hat{\Omega}^{+}$in which we are interested is a $\mathbb{Z}$-covering.

In a neighborhood of the complete inverse image $f^{-1}\left(M^{n}\right) \subset \hat{\hat{\Omega}}^{+}$, where $M^{n}$ a $\hat{\Omega}^{+}\left(M^{n}\right)$ is a set of one-point curves, we have a local minimum of the action $S_{E}$ on all sheets of the covering. Consider the image of a segment in $\hat{\Omega}^{+}$under the maps $\alpha_{j}: I \rightarrow \hat{\Omega}^{+}\left(M^{n}\right)$ such that the $\alpha_{j}(0)$ (the image of the origin) lie in the same set of one-point curves $M_{1}^{n} \subset \hat{\hat{\Omega}}^{+}\left(M^{n}\right)$, and the $\alpha_{j}(1)$ (the image of the end) lie in the other component $M_{2}^{n} \subset \hat{\hat{\Omega}}\left(M^{n}\right)$ of the complete inverse image $f^{-1}\left(M^{n}\right)$. We assume that $S_{E}=0$ on the "zero" component. Then the value of the action on the component $T_{j}\left(M_{1}^{n}\right)$ is equal to $\kappa_{j}, j=1, \ldots, k$ (we recall that the basis has been chosen so that $\kappa_{j}=0$ for $j>k$ ). The map $\alpha_{j}$ on $I$ generates a function with a maximum strictly inside the segment. By minimizing the maxima over all maps $\alpha_{j}$ 
we obtain the minimal map of the segment (a critical point of $S_{E}$ ). In the "general position" case this critical point has index 1 . The value $S_{E}\left(\gamma_{j}\right)$ of the action on this periodic extremal $\gamma_{j}$ is no less than $\kappa_{j}$. Thus, we have proved the following theorem.

Theorem 4. On a complete simply connected Riemannian manifold $M^{n}$ with a metric $g_{i j}$ there always exists a nontrivial (for any energy $E>0$ ) periodic extremal of any direction in a homologically nontrivial magnetic field $H_{i j}$. In the general position case the Morse index of this extremal is 1.

Now we consider an entirely similar situation but another problem: we look for extremals connecting the points $x_{1}$ and $x_{2}$. The simplest examples show that this problem is unsolvable even for $\mathbb{R}^{2}$ with the Euclidean metric in a one-dimensional (constant) magnetic field $H$ for a sufficiently small energy $E>0$. Let $x_{1}$ and $x_{2}$ be sufficiently close (for a given fixed energy $E$ ) that there is a locally minimal extremal $\gamma_{x_{1} x_{2}} \in \hat{\Omega}^{+}\left(M^{n}, x_{1}, x_{2}\right)$ joining them.

The proof of the following theorem is completely analogous to that of Theorem 4 .

Theorem 5. If for any complete simply connected Riemannian manifold $M^{n}$ with a distinguished 2-form $H$ there is at least one locally minimal (relative to $S_{E}$ ) extremal $\gamma_{x_{1} x_{2}}$ going from $x_{1}$ to $x_{2}$, then on $M^{n}$ there is at least one saddle extremal of index 1 with the same energy $E>0$, that goes from $x_{1}$ to $x_{2}$.

\section{Examples of Functionals with a Finite Symmetry Group}

In the theory of Kirchhoff equations the Poisson bracket (2) and the integrals $f_{1}$ and $f_{2}$ are invariant under all reflections $T_{k}$ in any of the axes, e.g.,

$$
\begin{gathered}
T_{k}: M_{i} \rightarrow-M_{i}, M_{j} \rightarrow-M_{j}, M_{k} \rightarrow M_{k}, p_{i} \rightarrow-p_{i}, p_{j} \rightarrow-p_{j}, \\
p_{k} \rightarrow p_{k}, \\
\sum M_{i} p_{i} \rightarrow \sum M_{i} p_{i}, \quad \sum p_{i}^{2} \rightarrow \sum p_{i}^{2}, \quad i \neq j \neq k .
\end{gathered}
$$

If under reflection (22) Hamiltonian (11) is mapped into itself, then this transformation enables us to decrease the number of closed trajectories in Theorem 1. In particular, the Hamiltonian $\left[(1), b_{i j}=b_{i i} \delta_{i j}, c_{i j}=c_{i i} \delta_{i j}\right]$ is invariant under the three reflections in the orthogonal planes (the integrals $f_{1}$ and $f_{2}$ are fixed) that generate the finite group of symmetries of $S_{E}$ on the space of directed curves. In principle, we can consider Hamiltonians $H$ invariant under any finite subgroups $G \subset S O(3)$. But for quadratic Hamiltonians of the form (1), the presence in $G$ of at least one element of order greater than 2 automatically makes the problem trivial (it gives rise to a continuous group of symmetries and complete integrability).

Example 1. The group $G=\mathbb{Z}_{2}$ (a reflection in an axis; let $k=3$ ). Then the following elements are trivial: $a_{i 3}=b_{i 3}=c_{i 3}=0(i \neq 3)$. The transformation $T_{3}$ generates a transformation $T_{3}^{*}$ of the space of closed non-self-intersecting directed curves into itself. All the fixed points $T_{3}^{*} \gamma=\gamma$ of $T_{3}^{*}$ are plane sections of the sphere $S^{2}$ (the integrals $s$ and $p$ are fixed) perpendicular to the third axis with a given direction. The transformation $T_{3}^{*}$ does not change the direction of the curve $\gamma$. Therefore, the set $Q$ of all fixed points $T_{3}^{*} \gamma=\gamma$ is the union of two intervals with endpoints resting on one-point curves:

$$
Q=Q^{+} \cup Q^{-} \subset \hat{\Omega}_{1}^{+}\left(S^{2}\right), \quad T_{3}^{*} Q^{+}=Q^{+}, \quad T_{2}^{*} Q^{-}=Q^{-},
$$


S. P. NOVIKOV

where $\hat{\Omega}_{1}^{+}$is the space of non-self-intersecting directed curves. The following obvious but useful result holds.

Lemma 6. The gradient of any function invariant under a finite group of transformations (in a metric also invariant under the same group) is tangential to the set of fixed points.

The values of $S_{E}$ on both components of the boundary $\partial Q^{+}=\partial Q^{-}$can be different (one of them is zero) (the boundary consists of one-point curves), and $\delta^{2} S_{E}>0$ in a small neighhorbood of the one-point curves. Therefore, there is not less than one maximum of the function $S_{E}$ bounded on each of the submanifolds $Q^{+}, Q^{-}$, or not less than two critical points of index not less than 1 in the surrounding space $\hat{\Omega}_{1}^{+}\left(S^{2}\right)$ for every energy $E>\max U=U_{0}$.

We consider the general position case when all the critical points are nondegenerate. In the space $\hat{\Omega}_{1}^{+}\left(S^{2}\right) \times\left(\bmod K_{0}\right)$ (of one-point curves) there are two cycles (one- and three-dimensional). It is possible to have critic points $\tilde{\gamma}_{j}$ outside $Q$, but then a number of them must be repeated $\tilde{\gamma}_{j} \neq T_{3}^{*} \tilde{\gamma}_{j}$; here $\tilde{\gamma}_{j}$ and $T_{3}^{*} \tilde{\gamma}_{j}$ have the same Morse index. From here we obtain the following assertion.

Assertion. Among the plane sections of the sphere $S^{2}$ orthogonal to an axis of symmetry there is at least one periodic extremal for each direction of motion, the Morse indexes of which are not equal to each other, and the value of $S_{E}$ on each is positive $\left.\left(S_{E}\right)\left(\gamma^{ \pm}\right)>4 \pi|s|\right)$.

Example 2. Let $a_{i j}=a_{i i} \delta_{i j}, b_{i j}=b_{i i} \delta_{i j}$, and $c_{i j}=c_{i i} \delta_{i j}$. Then the problem admits the group $G=\mathbb{Z}_{2} \times \mathbb{Z}_{2}$. All three nonzero elements of $G$ are simply reflections with respect to the orthogonal axes $T_{1}, T_{2}$ and $T_{3}=T_{1} T_{2}$ with $T_{i}^{2}=1$. We apply the preceding argument to all three transformations $T_{i}^{*}$ that preserve the value of $S_{E}$. For $E>\max U=U_{0}$ there are periodic extremals of the type of plane sections of the sphere on all sets of fixed $T_{i}^{*} Q_{i}^{ \pm}=Q_{i}^{ \pm}, i=1,2,3$, for both directions of motion (in all we obtain not less than six extremals for which $S_{E}\left(\gamma_{i}^{ \pm}\right)>4 \pi|s|$ ).

\section{APPENDIX}

\section{LegGett's Equation for the Spin in the Superfluid ${ }^{3}$ He}

The homogeneous states of the superfluid ${ }^{3} \mathrm{He}$ in the so-called $A$ and $B$ phases are described by a complex $(3 \times 3)$ matrix $A_{i j}$, i.e., by a point of one of the matrix manifolds $M_{A}$ or $M_{B}$ :

1) $A$ phase: $A_{j k}=($ const $) \cdot d_{j} \cdot\left(\Delta_{k}^{\prime}+i \Delta_{k}^{\prime \prime}\right),|d|^{2}=\left|\Delta^{\prime}\right|^{2}=\left|\Delta^{\prime \prime}\right|^{2}=1, \Delta^{\prime} \Delta^{\prime \prime}=0$;

2) $B$ phase: $A_{j k}=($ const $) \cdot e^{i \phi} \cdot R_{j k}(\theta, \hat{n})$, where $R_{i j} \in S O_{3}, \hat{n}$ is the direction of an axis, and $\theta$ is an angle of rotation about the $\hat{n}$ axis.

In a state with nonzero spin $s=\left(s_{1}, s_{2}, s_{3}\right)$ in a magnetic field $B^{i}$, the energy has the form

$$
H=\frac{1}{2} g^{2} \chi^{i j} s_{i} s_{j}-g B^{i} s_{i}+V\left(A_{j k}\right)
$$

where $g=$ const, $x^{i j}$ is the magnetic susceptibility tensor depending possibly on $A_{j k}$, and $V=V\left(A_{j k}\right)$ with $A_{j k} \in M_{A}$ or $A_{j k} \in M_{B}$.

In the $A$ phase case the tensor $\chi^{i j}(d)$ usually has the form

$$
\chi_{A}^{i j} s_{i} s_{j}=\chi^{-1}\left(\sum s_{i}^{2}\right)+(\text { const })\left(\sum s_{i} d_{i}\right)^{2}
$$


For the $B$ phase case we assume the susceptibility tensor to be isotropic

$$
\chi_{B}^{i j}=\chi^{-1} \delta^{i j}, \quad \chi=\text { const. }
$$

The potential $V$ (a dipole-dipole interaction) has the form

$$
V=V_{A}(d)=-c_{1}^{2}\left(l^{i} d_{i}\right)^{2}
$$

in the $A$-phase case, and

$$
V=V_{B}(\cos \theta)=c_{2}^{2}\left(\frac{1}{2}+2 \cos \theta\right)^{2}
$$

for the $B$ phase, where $1+2 \cos \theta=S p\left(R_{j k}\right)=R_{j j}$. The vector $l$ is assumed to be constant, and $c_{1}$ and $c_{2}$ are certain constants. Note that for the $A$ phase the potential $V_{A}$ does not depend on the "orbital" variables $\Delta^{\prime}$ and $\Delta^{\prime \prime}$. The equations of motion will be defined only for the pair $(s, d)$ in the $A$-phase case. In the $B$-phase case we consider an energy independent of the velocity potential $\phi$, and shall discuss the equations only in a state of hydrodynamic rest for the variables $\left(s_{i}, R_{j k}\right)$. As Golo, Fomin, and Volovik have remarked to the author, Leggett's equations for the $A$ and $B$ phases (neglecting viscosity) can be obtained from the Hamiltonian (1) with respect to the following Poisson brackets:

$$
\text { 1) }\left\{s_{i}, s_{j}\right\}=\varepsilon_{i j k} s_{k}, \quad\left\{s_{i}, d_{j}\right\}=\varepsilon_{i j k} d_{k}, \quad\left\{d_{i}, d_{j}\right\}=0
$$

for the $A$ phase. Here $d^{2}=1$ or $d \in S^{2}$.

$$
\text { 2) }\left\{s_{i}, s_{j}\right\}=\varepsilon_{i j k} s_{k}, \quad\left\{R_{l m}, R_{s t}\right\}=0, \quad\left\{s_{i}, R_{j m}\right\}=\varepsilon_{i j k} R_{k m}
$$

for the $B$ phase. Here $R_{j k}(\theta, \hat{n}) \in S O_{3}$, where

$$
\hat{n}^{2}=1, \quad R_{j k}=\cos \theta \delta_{j k}+(1-\cos \theta) \hat{n}_{j} \hat{n}_{k}+\sin \theta \varepsilon_{i j k} \hat{n}_{i} .
$$

The Poisson brackets $\left(5^{\prime}\right)$ for the $A$ phase correspond to the Lie algebra of $E(3)$; the Hamiltonian (1) has a form satisfying the conditions of Theorems 1 and 2 above. Thus, we obtain the following conclusion: all the results of the preceding sections about periodic orbits remain valid for Leggett's equations in the $A$ phase, provided that viscosity is neglected. If we can regard viscosity as small (i.e., the influence of the viscosity term on the period of rotation along an orbit is small), then we obtain periodic orbits of a conservative system that are slowly losing energy as a result of viscosity. We recall that we have proved the existence of periodic orbits for all energy values greater than a threshold of the potential, and for all values of the "Kirchhoff integrals" $d^{2}=1,(s, d)=$ const.

Now we turn to the $B$ phase. The Poisson bracket $\left(5^{\prime \prime}\right)$ is simply the usual Poisson bracket on $T^{*}\left(S_{3}\right)$ in the variables $R \in S_{3}$ and $s^{*}=\dot{R} R^{-1}$, which give a representation in the form of a direct product $T^{*}\left(\mathrm{SO}_{3}\right)=\mathbb{R}^{3} \times \mathrm{SO}_{3}$. Thus, Leggett's equation for the $B$ phase in a magnetic field can be obtained from a Lagrangian system on $\mathrm{SO}_{3}$. In view of results in Sec. 4 we establish the existence of periodic orbits, at least one for each energy greater than threshold $E>\max V_{B}(R)$. We can also obtain an analogue of the result in Sec. 1 about the appearance of a small "saddle" orbit $\left[E \sim \max V_{B}(R)\right]$ for magnetic fields greater than some threshold. But this is too "small."

Leggett's system for the $B$ phase in a zero magnetic field is exactly integrable as was proved by Mackey and Ebisava in 1976 (see the survey by Brinkman and Cross in [3]). In a nonzero magnetic field $B \neq 0$, Leggett's system has only one 
redundant integral. Therefore, we must look for periodic orbits that depend on two continual parameters - energy and the value of the integral (for $B \rightarrow \infty$ see [4]).

For a nonzero magnetic field the Hamiltonian $H(s, R)$ has the form $(1),(3)$. We consider a general Hamiltonian of the form

$$
H=\frac{1}{2} s^{2}+V(\cos \theta)
$$

where $V$ is an arbitrary function.

Lemma. The system generated by the Hamiltonian (6) with the Poisson bracket $\left(5^{\prime \prime}\right)$ is always completely integrable.

Proof. In fact, by definition we have

a) the quantity $1+2 \cos \theta=\operatorname{Sp} R=R_{i j}$ is invariant under inner automorphisms;

b) the Killing metric $s^{2}$ on the group $\mathrm{SO}_{3}$ is invariant under inner automorphisms (it is invariant under all right and left translations).

As a consequence of this, the Hamiltonian (6) and the corresponding Lagrangian on $\mathrm{SO}_{3}$ are invariant under all inner automorphisms

$$
s \rightarrow g s^{\prime}, \quad s \in \mathbb{R}^{3}, \quad R \rightarrow g R g^{-1}, \quad g \in S O_{3}, R \in S O_{3} .
$$

The group of symmetries [the transformations (7)] generates the invariant vector

$$
A=(1-\cos \theta)\left[\hat{n} \times\left(\operatorname{ctg} \frac{\theta}{2} s+[\hat{n} \times s]\right)\right]
$$

where $R_{j k}=(1-\cos \theta) \hat{n}_{j} \hat{n}_{k}+\cos \theta \delta_{j k}+\sin \theta \varepsilon_{i j k} \hat{n}_{i}, \theta$ is an angle of rotation about the $\hat{n}$ axis and $\sum \hat{n}_{i}^{2}=1$. This vector is given by Brinkman and Cross [3] as a result of a direct formal conjecture.

In the work of Mackey and Ebisava (1976) (see [3]), where this problem is integrated in a zero magnetic field, symmetry considerations are not discussed.

The Poisson brackets of the components of the vector $A$ and the Hamiltonian are zero:

$$
\left\{A_{i}, H\right\}=0 .
$$

Since $A_{i}$ is a generator of the group $\mathrm{SO}_{3}$ which acts by means of the representation (7), we have the Poisson brackets

$$
\left\{A_{i}, A_{j}\right\}=\varepsilon_{i j k} A_{k}, \quad\left\{A_{i}, A^{2}\right\}=0
$$

(although the $A_{i}$ are not moments of momentum). As a consequence of this, system (6) is integrable (the integrals $H, A_{1}$ and $A^{2}$ commute). Moreover, the level surfaces of the four integrals $\left(H, A_{1}, A_{2}, A_{3}\right)$ in general position are two-dimensional tori. This proves the lemma.

In a nonzero magnetic field $\left(B^{i}\right)$ directed along the first axis $\left(B^{1}=B, B^{2}=\right.$ $\left.B^{3}=0\right)$, Leggett's Hamiltonian reduces to the form

$$
H=\frac{s^{2}}{2}+\lambda s_{1}+V(\cos \theta) .
$$

In a constant magnetic field there is as well as the energy $H=E$ another integral $A_{1}=$ (const) $\cdot A_{i} B^{i}$ generated by the action (7) of the group of rotations around the field

$$
A_{1}=-a \sin \theta+(1-\cos \theta)\left(s_{1}-s_{\|} \hat{n}_{1}\right)
$$


where

$$
a=s_{2} \hat{n}_{3}-\hat{n}_{2} s_{3}, \quad s_{\|}=s_{i} \hat{n}_{i} .
$$

By using the integral $A_{1}$ we can "factorize the Hamiltonian formalism" (or the Lagrangian) and go over a configuration space of the form $\mathrm{M}^{2}=\mathrm{SO}_{3} / \mathrm{SO}_{2}$ (not to be confused with a Hopf fibering). We recall that tbe action of $\mathrm{SO}_{2}$ on $\mathrm{SO}_{3}$ has the form (7)

$$
R \rightarrow g R g^{-1}, \quad g \in S O_{2}
$$

(here $g$ is a rotation about the first axis - the direction of the magnetic field $B$ ). The situation here differs sharply from the $A$ phase, the Kirchhoff problem, and the classical top in an axisymmetric gravitational field: in the top problem the factorization of the configuration space $\mathrm{SO}_{3}$ is performed [2] by using right translations from the subgroup of symmetries $\mathrm{SO}_{2}$, and always gives a Hopf fibering with a base that is a two-dimensional sphere without singularities. In this case we obtain a factor $M^{2}=\left(\right.$ a disk $D^{2}$ with a boundary $\left.\Gamma=S^{1}\right)$; the boundary contains singular points. It is technically convenient to calculate the factor-system on $M^{2}$ in the following way.

The quantities $\left(s^{2}, s_{\|}, \theta, s_{1}, n=\hat{n}_{1}, a=[s \times \hat{n}]_{1}=s_{2} \hat{n}_{3}-\hat{n}_{2} s_{3}\right)$ are invariant under the transformations (14). In addition, there is a simple connection that follows from elementary geometry

$$
s^{2} a^{2}=\left(s^{2}-s_{1}^{2}\right)\left(s^{2}-s_{\|}^{2}\right)-\left(s^{2} n-s_{1} s_{\|}\right)^{2} .
$$

Thus, the space of parameters $\left(s^{2}, s_{\|}, s_{1}, n, a, \theta\right)$ is five-dimensional. Consequently, there is a function of these parameters such that its Poisson bracket with all of them vanishes. ${ }^{6}$ This function is $A_{1}$ :

$$
\begin{aligned}
A_{1} & =A_{1}\left(\theta, a, s_{1}, s_{\|}, n\right), \\
\left\{A_{1}, s^{2}\right\}=\left\{A_{1}, s_{\|}\right\} & =\left\{A_{1}, \theta\right\}=\left\{A_{1}, n\right\}=\left\{A_{1}, s_{1}\right\}=0 .
\end{aligned}
$$

Deduction. Under the condition $A_{1}=$ const we can use the earlier formulas for the paired Poisson brackets of these parameters.

Simple calculations starting from $\left(5^{\prime \prime}\right)$ give the following result:

$$
\begin{gathered}
\left\{s_{\|}, \theta\right\}=1, \quad\{\theta, n\}=0, \quad\left\{f, s^{2}\right\}=0, \quad\{f, \theta\}=0, \quad\left\{f, \sigma_{\|}\right\}=0, \\
\{f, n\}=\sqrt{\frac{1}{2}\left(1-n^{2}\right) f-\frac{A_{1}^{2}}{4}}, \quad f=\left(s^{2}-s_{\|}^{2}\right)(1-\cos \theta)=\frac{1}{2} \sum A_{i}^{2}=\frac{1}{2} A^{2} .
\end{gathered}
$$

Thus, on the level surface $A_{1}$ the Poisson bracket (a 2-form) reduces to a canonical form with the coordinates

$$
\begin{gathered}
\left(\theta, n, p_{\theta}, p_{n}\right), \quad \Omega=d \theta \wedge d p_{\theta}+d n \wedge d p_{n}, \quad p_{\theta}=s_{\|} \\
p_{n}=\sqrt{\frac{2 f}{1-n^{2}}-\frac{A_{1}^{2}}{\left(1-n^{2}\right)^{2}}}
\end{gathered}
$$

\footnotetext{
${ }^{6}$ The closed subalgebra of the Poisson brackets of $\left(s^{2}, s_{\|}, \theta\right)$ was first discussed by V. L. Golo. The collection of all parameters (15) gives a closed algebra of brackets.
} 
Hence, we obviously have

$$
\begin{gathered}
s^{2}=p_{\theta}^{2}+\frac{1-n^{2}}{2(1-\cos \theta)}\left(p_{n}^{2}+\frac{A_{1}^{2}}{\left(1-n^{2}\right)^{2}}\right) \\
H=\frac{s^{2}}{2}+\lambda s_{1}+V(\cos \theta), \quad 2 f=\left(1-n^{2}\right) p_{n}^{2}+A_{1}^{2} /\left(1-n^{2}\right) \\
s_{1}=n p_{\theta}+\frac{1-n^{2}}{2} \sin \theta p_{n}+A_{1} \frac{2-\sin ^{2} \theta}{2(1-\cos \theta)}
\end{gathered}
$$

By using (18) and (19) we obtain the following result in the variables $\chi=\theta / 2$ and $n=\sin \phi$.

Theorem. Leggett's system in the B phase is equivalent to a Lagrangian system, viz., a charged particle on the sphere $S^{2}$ in an effective magnetic field. The Lagrangian has the form

$$
L=2\left(\dot{\chi}^{2}+\sin ^{2} \chi \dot{\phi}\right)-A_{\chi} \dot{\chi}-A_{\phi} \dot{\phi}-U(\chi, \phi)
$$

where

$$
\begin{gathered}
A_{\chi}=2 \lambda \sin \phi, \quad A_{\phi}=8 \lambda \cos \phi \sin ^{3} \chi \cos \chi \\
U=V(\cos \theta)+\frac{A_{1}^{2}}{4 \sin ^{2} \chi \cos ^{2} \phi}+\frac{\lambda A_{1}\left(1-\sin ^{2} \chi \cos ^{2} \chi\right)}{2 \sin ^{2} \chi} \\
-\frac{\lambda}{2}\left(\sin ^{2} \phi+4 \cos ^{2} \phi \sin ^{3} \chi \cos \chi\right) .
\end{gathered}
$$

The proof is given above.

For any $A_{1} \neq 0$ the system has singularities on a great circle of the sphere:

$$
\phi= \pm \pi / 2
$$

and stronger singularities at the poles

$$
\chi=0, \pi \text {. }
$$

Thus, when $A_{1} \neq 0$ we always have a system on a manifold with an edge (a domain on $S^{2}$ )

$$
U(\chi, \phi) \leqslant E .
$$

This domain lies inside a great circle

$$
0<\chi<\pi, \quad-\pi / 2<\phi<\pi / 2
$$

and is at a nonzero distance from the boundary.

For $A_{1}=0$ the system is defined and is regular on the whole sphere $S^{2}$ except for the poles $\chi \neq 0, \pi$, where the "effective magnetic field" and potential have singularities.

If the magnetic field is zero (here $\lambda=0$ ), then we have the integral $2 f=$ $\left(4 \sin ^{2} \chi \dot{\phi}\right)^{2}+A_{1}^{2} \cos ^{-2} \phi$, which enables us at last to integrate the system.

In a zero magnetic field the system seems to be nonintegrable. A certain set of periodic solutions of the original Leggett system $(1),(4),(5)$ gives stationary solutions of the factorized system in the coordinates $\left(\theta, n, p_{\theta}, p_{n}\right)$ with the Hamiltonian (19). For the condition for the factor-system to be stationary means that in the original coordinates $\left(s_{i}, R_{j k}\right)$ the solution is periodic and coincides with an orbit of the group of symmetries - a 1-parameter group of rotations of the field that acts on $\mathrm{SO}_{3}$ by the inner automorphisms (7). 


\section{REFERENCES}

[1] S. P. Novikov and I. Shmel'tser, "Periodic solutions of the Kirchhoff equation for the free motion of a rigid body in an ideal incompressible fluid, and the extended Lyusternik-ShnirelmanMorse theory. I," Funkts. Anal. Prilozhen., 15, No. 3, 54-66 (1981).

[2] V. V. Kozlov, Methods of Qualitative Analysis in the Dynamics of a Rigid Body [in Russian], Moscow State Univ. (1980).

[3] W. F. Brinkman and M. C. Cross, in: Progress in Low Temperature Physics (ed. D. F. Brewer), Oxford Univ. Press, Oxford-New York-Amsterdam, North-Holland, Amsterdam (1978), pp. 105-198.

[4] I. A. Fomin, "The NMR in the superfluid ${ }^{3}$ He," J. Low Temp. Phys., 31, Nos. 3/4, 509-526 (1978). 